\title{
Recovered or Resolved with Sequelae
}

National Cancer Institute

\section{Source}

National Cancer Institute. Recovered or Resolved with Sequelae. NCI Thesaurus. Code C49495.

One of the possible results of an adverse event outcome where the subject recuperated but retained pathological conditions resulting from the prior disease or injury. 